\title{
BMJ Open Quality Enhancing the performance of gastrointestinal tumour board by improving documentation
}

\author{
Roaa Saleh Alsuhaibani, ${ }^{1}$ Hajer Alzahrani, ${ }^{2}$ Ghada Algwaiz, ${ }^{2}$ Haneen Alfarhan, ${ }^{2}$ \\ Ashwaq Alolayan, ${ }^{3}$ Nafisa Abdelhafiz, ${ }^{3}$ Yosra Ali, ${ }^{3}$ Abdul Rahman Jazieh ${ }^{3}$
}

To cite: Alsuhaibani RS, Alzahrani H, Algwaiz G, et al. Enhancing the performance of gastrointestinal tumour board by improving documentation. BMJ Open Quality 2018;7:e00168. doi:10.1136/

bmjoq-2017-000168

- Additional material is published online only. To view please visit the journal online (http://dx.doi.org/10.1136/ bmjoq-2017-000168).

Received 20 July 2017 Revised 6 February 2018 Accepted 9 February 2018

\section{Check for updates}

${ }^{1}$ College of Medicine, King Saud bin Abdulaziz University for Health Sciences, Riyadh, Saudi Arabia

${ }^{2}$ College of Medicine, King Saud bin Abdulaziz University for Health Sciences, Riyadh, Saudi Arabia

${ }^{3}$ Department of Oncology, King Abdulaziz Medical City, Riyadh, Saudi Arabia

Correspondence to Dr Abdul Rahman Jazieh; jazieha@ngha.med.sa

\section{ABSTRACT}

Tumour board contributes to providing better patient care by using a multidisciplinary team approach. In the efforts of evaluating the performance of the gastrointestinal tumour board at our institution, it was difficult to assess past performance due to lack of proper use of standardised documentation tool. This project aimed at improving adherence to the documentation tool and its recommendations in order to obtain performance measures for the tumour board. A multidisciplinary team and a plan were developed to improve documentation. Four rapid improvement cycles, Plan-Do-Study-Act (PDSA) cycles, were conducted. The first cycle focused on updating the case discussion summary form (CDSF) based on experts' input and previous identified deficiencies to enhance documentation and improve performance. The second PDSA cycle aimed at incorporating the CDSF into the electronic medical records system and assessing its functionality. The third cycle was to orient and train staff on using the form and launching it. The fourth PDSA cycle aimed at assessing the ability to obtain tumour board performance measures. Adherence to completion of the CDSF improved from $82 \%$ (baseline) to $94 \%$ after the fourth PDSA cycle. Over 104 consecutive cases discussed in the tumour board between January and July 2016 and 76 cases discussed in 2015, results were as follows: adherence to National Comprehensive Cancer Network guidelines in 2016 was observed in 141 (95\%) recommendations, while it was observed in $90(92 \%)$ recommendations in 2015 . Changes in the management plans were observed in $37(36 \%)$ cases in 2016 and in $6(8 \%)$ cases in 2015 . Regarding tumour board recommendations, $87 \%$ were done within 3 months of tumour board discussion in 2016, while $69 \%$ were done in 2015. Implementing electronic standardised documentation tool improved communication among the team and enabled getting accurate data about performance measures of the tumour board with positive impact on healthcare process and outcomes.

\section{PROBLEIM}

The aim of a gastrointestinal (GI) tumour board is to discuss management plans for oncological cases by specialised multidisciplinary teams and decide on the optimal plan for each case. An active multidisciplinary GI tumour board is held on a weekly basis for years. However, evaluating the performance and the impact of GI tumour board was difficult due to lack of proper identified performance measures. Among the possible identified causes of this problem was lack of proper use of a standardised documentation tool by the GI tumour board team. In addition, non-compliance to completion of the case discussion summary form (CDSF) was noted among the GI tumour board team, which made the evaluation of GI tumour board performance more difficult.

\section{BACKGROUND}

In recent years, physicians have realised the importance of different specialties working more closely with each other to enhance the care provided to patients. This concept is essential for cancer patients especially due to the complexity of the treatment. Tumour boards play a vital role as a multidisciplinary team and they are recommended in the literature and by national and subspecialty guidelines. ${ }^{1}$ Measuring the quality of the output from tumour boards, such as the adherence of recommendation to guidelines and ensuring the recommendations are done, would be essential to ensure maximum productivity and benefit for patients. In the efforts to evaluate the performance of GI tumour boards, it was difficult to assess past performance due to lack of proper use of a standardised documentation tool. Establishing a highquality tumour board requires adequate and efficient documentation. ${ }^{2}$ As perceived by the American College of Surgeons Commission on Cancer, the Rapid Quality Reporting System applied on the breast multidisciplinary teams increased the rate of adherence to guidelines. ${ }^{3}$ Having records of the tumour board will allow the GI tumour board team to analyse and identify areas of improvement and raise the quality of the meetings. As GI cancers are very common malignancies in Saudi Arabia, ${ }^{4}$ it is crucial to provide 
the best care to this large population by improving the performance of the GI tumour board. This goal cannot be achieved without good adherence to tumour board recommendations and the documentation tool. Hence, this project aimed at improving adherence to the documentation tool in order to obtain performance measures of the GI tumour board.

\section{BASELINE MEASUREMENT}

Data from 13 consecutive tumour boards that were held in the first quarter of 2015 were collected retrospectively from the medical records using a validated data collection form. A total of 76 cases were presented to the tumour board at this period. Adherence to completion of the CDSF was $82 \%$. Adherence to National Comprehensive Cancer Network (NCCN) guidelines was observed in 90 recommendations (92\%). Changes in the management plans were observed in six cases $(8 \%)$. Recommendations that were done within 3 months of tumour board discussion accounted for $69 \%$ of the recommendations.

\section{DESIGN}

A multidisciplinary team was formed including oncology, surgery, pathology and radiology physicians with data management. The team developed a plan to improve the documentation in the GI tumour board to facilitate communication among team members. The plan was to use rapid improvement Plan-Do-Study- Act (PDSA) cycles in developing, implementing and evaluating the intervention.

\section{STRATEGY}

Four rapid improvement PDSA cycles were applied to the GI tumour board in the period between 2015 and 2016. PDSA cycle 1: Our initial intervention was to update the CDSF that is used to document the discussion findings in the weekly GI tumour board. Updates were based on team members' input and previously identified deficiencies in the form. Updates included classifying the recommendations based on the type of management patients would receive. A section regarding implementation of the GI tumour board recommendations within the following 3 months was added (online supplementary 1). After that, the modified CDSF was applied in the following GI tumour boards. Feedback from GI tumour board participants stated that these updates would be more effective if we move them to an electronic form. PDSA cycle 2: We hypothesised that our change idea will be more effective if we incorporate the CDSF into the electronic medical record system and assess its functionality. Implementation of the CDSF was achieved by the assistance of the Information Technology Department. The electronic CDSF was tested by three consultants on a small scale before generalising it to all GI tumour board members. Since the results and the consultants' feedback were encouraging, the electronic CDSF was adopted. PDSA cycle 3: We aimed to orient and train staff on using the electronic CDSF routinely. GI tumour board members were trained and their compliance was assessed. After observing sufficient compliance by GI tumour board members, the electronic CDSF totally replaced the paper CDSF. PDSA cycle 4: Intended mainly to assess our ability to obtain GI tumour board performance measures. Performance measures included the adherence to the documentation tool and completion of the CDSF, adherence of GI tumour board recommendations to NCCN guidelines, adherence of physicians to GI tumour board recommendations and changes on patients' management plans. Performance measures were collected prospectively (weekly from the GI tumour board) using a data collection form that was developed by the primary investigator and tested several times before. By comparing baseline measurements of 2015 with 2016 measurements, after completion of all PDSA cycles, the results were satisfying and encouraging changes were observed in the performance. We standardised the GI tumour board performance measures and continuously monitored the performance and the compliance to documentation. Weekly feedback from GI tumour board members is collected and weekly submitted electronic CDSFs are reviewed by the oncology department to ensure adherence to completion of CDSFs and sustainability of performance.

\section{RESULTS}

Among all collected GI tumour board cases in 2016, the adherence of completion of CDSF was observed in $94 \%$ of the cases, while it was observed in $82 \%$ of the cases in 2015. As for tumour board recommendations, 149 recommendations were made in 2016, while 105 were made in 2015. Out of that number, adherence to NCCN guidelines in 2016 was observed in 141 recommendations $(95 \%)$, while it was observed in 90 recommendations $(92 \%)$ in 2015 (table 1). The justification for not following the NCCN guidelines were explained by difficulty of performing the recommended treatment plan for the patient due to poor health status, no response to guideline choice of treatment or patient preference and physician consensus. Alternative treatment options

Table 1 Main results of 180 cases discussed in tumour board between 2015 and 2016

\begin{tabular}{lcc}
\hline Variables & 2015 n (\%) & $\mathbf{2 0 1 6}$ n (\%) \\
\hline No of GITB recommendations & 105 & 149 \\
Adherence to NCCN guidelines & $90(92 \%)$ & $141(95 \%)$ \\
$\begin{array}{l}\text { Completion of GITB } \\
\text { recommendations }\end{array}$ & $68(69 \%)$ & $130(87 \%)$ \\
Impact on management & $6(8 \%)$ & $37(36 \%)$ \\
Follow-up in next GITB & $4(5 \%)$ & $7(7 \%)$ \\
\hline
\end{tabular}

GITB, gastrointestinal tumour board; NCCN, National Comprehensive Cancer Network. 


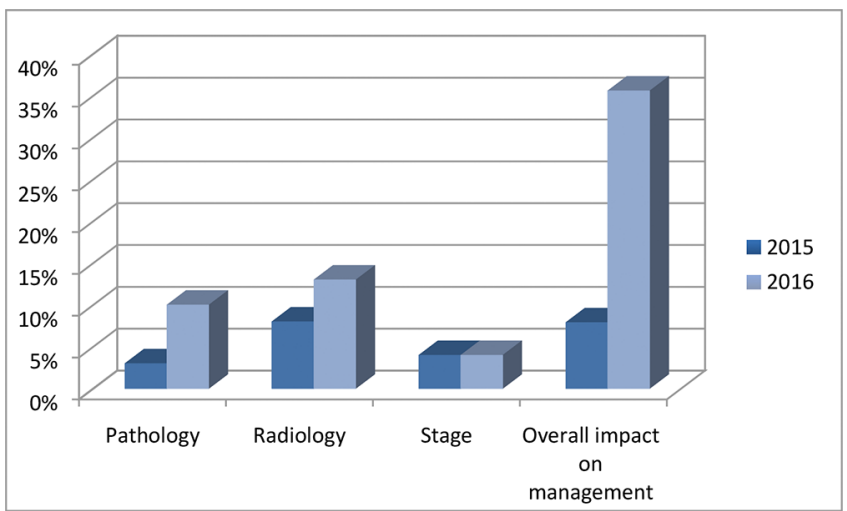

Figure 1 Impact of gastrointestinal tumour board on pathology, radiology, staging and management.

were based on treating physician's judgement, tumour board discussion of the case and patient's preference. Furthermore, 130 recommendations $(87 \%)$ were done by treating physicians within the next 3 months following GI tumour board in 2016, while 68 recommendations $(69 \%)$ were done in 2015 (table 1).

During the tumour board meetings, new findings were revealed in pathology and radiology, which led to a change in staging and impacted overall management of discussed cases (figure 1). The radiological and pathological findings included discovery of presence or absence of lymph nodes, change in tumour size, changes in histological type of cancer and evidence of metastasis.

Out of 104 cases in 2016, GI tumour board impacted the management of 37 cases $(36 \%)$, while impacts on management were observed in 6 cases $(8 \%)$ in 2015. The impact included addition of adjuvant or neoadjuvant chemotherapy, addition of radiotherapy, changes in chemotherapy regimen, discontinuation of chemotherapy, re-staging, observation, surgery, transplant, ultrasound or biopsy. Follow-up in next GI tumour board to follow updates in radiological/pathological findings and effect of treatment on patients were recommended for seven cases $(7 \%)$ in 2016 while it was recommended for four cases $(5 \%)$ in 2015 (table 1).

\section{LESSONS AND LIMITATIONS}

The project aim was to improve the documentation of the tumour board in order to improve performance, with the key focus of implementing an electronic documentation tool. For this to be achieved, a modified form needed to be designed that would suit various cases. Building on previous ideas, we also wanted an electronic system that offers a more accommodating and secure place for CDSF to be documented and stored in. A key lesson learnt during the process was the importance of PDSA cycles, which helped us to ensure that each step is built on the success of the previous step. These PDSA cycles acted as a test method of the ideas generated by the team and also were educational opportunities to the participants involved in the GI tumour board. Educating the member about the importance of the project, the importance of better documentation and compliance with the completion of the form were essential components of the success of the project. As enhancing multidisciplinary communication lay at the essence of the project, we ensured that all participants (oncologists, radiologists, surgeons and pathologists) were involved throughout the process by attending tumour board meetings, collecting written feedback and by individual training. However, despite all previous effort to emphasise the importance of proper accurate documentation, we noticed some poor documentation of CDSF by some participants. This issue highlights the fact that creating an electronic form does not guarantee by itself the resolution of communication challenge. Feedback to team members and constant monitoring should be conducted to evaluate proper use of the forms on a continuous basis. Assuring appropriate orientation about the form to the new tumour board members, such as new staff or rotating residents, is important to help them complete the documentation properly.

Also, one of the limitations of this study is the small sample size. Having a larger number of cases may allow us to evaluate the performance of the GI tumour board and detect any deficiency in the form more precisely. Furthermore, in our study, we exclusively studied the GI tumour board, which may not reflect the performance of other tumour boards in other specialties. For this to be improved further, we plan to include other tumour boards in all different specialties in the dissemination phase of the plan. For GI tumour board, the electronic CDSF became a permanent part of the workflow of the team to assure sustainability. Recognising that implementing electronic forms in the work process is important for sustainability, but it is not sufficient to assure compliance of staff. Therefore, continuous monitoring of performance with feedback to team members is critical to recognise any deficiency in documentation and work on corrective course of actions. Finally, assessing the impact of this documentation on the actual outcomes of patients such as improving survival or other outcome measures would be of great value to consider in future studies.

\section{CONCLUSION}

Implementing electronic standardised documentation tool improved communication among the team and enabled the team to get accurate data about performance measures of the tumour board. Based on this study, we recommend continuing using the current electronic CDSF and we are encouraging other tumour boards to implant the electronic CDSF.

Acknowledgements This work was made possible by the generous cooperation and support from Dr Sami Boghdadly, Department of General Surgery, King Abdulaziz Medical City, Ministry of National Guard Health Affairs, Riyadh.

Contributors RSA participated in data collection for the whole trial, drafted and revised the paper. HAlz, GA and HAlf participated in data collection and revising the 
manuscript. NA and AA monitored data collection and revised the draft paper. YA designed the data collection tool, wrote the statistical analysis plan and analysed the data. ARJ initiated the project, designed the data collection tool, implemented the project for the oncology department, monitored data collection for the whole trial and revised the draft paper.

Funding This research received no specific grant funding agency in the public, commercial or not-for-profit sectors.

Competing interests None declared.

Provenance and peer review Not commissioned; externally peer reviewed.

Data sharing statement № additional data are available.

Open Access This is an Open Access article distributed in accordance with the Creative Commons Attribution Non Commercial (CC BY-NC 4.0) license, which permits others to distribute, remix, adapt, build upon this work non-commercially, and license their derivative works on different terms, provided the original work is properly cited and the use is non-commercial. See: http://creativecommons.org/ licenses/by-nc/4.0/
C Published by the BMJ Publishing Group Limited. For permission to use (where not already granted under a licence) please go to http://www.bmj.com/company/ products-services/rights-and-licensing/

\section{REFERENCES}

1. El Saghir NS, Keating NL, Carlson RW, et al. Tumor boards: optimizing the structure and improving efficiency of multidisciplinary management of patients with cancer worldwide. Am Soc Clin Oncol Educ Book 2014:e461-e466.

2. Jalil R, Akhter W, Lamb BW, et al. Validation of team performance assessment of multidisciplinary tumor boards. J Urol 2014;192:891-8.

3. Farrugia DJ, Fischer TD, Delitto D, et al. Improved breast cancer care quality metrics after implementation of a standardized tumor board documentation template. J Oncol Pract 2015;11:421-3.

4. Mehmood A, Urcia J, Khan A, et al. Tumor registry annual report. Riyadh: King Faisal Specialist Hospital and Research Centre, 2013. (cited 14 Sep 2014). 